\title{
Total synthesis of splenocin B, a potent inhibitor of the pro-inflammatory cytokine from marine-derived Streptomyces sp.
}

\author{
Ken-ichi Yoshida, Minako Ijiri, Hideo Iio and Yoshinosuke Usuki* \\ Division of Molecular Materials Science, Graduate School of Science, Osaka City University, 3-3-138 Sugimoto, Sumiyoshi-ku, Osaka 558-8585, Japan
}

\section{ARTICLE INFO ABSTRACT}

Article history:

Received

Received in revised form

Accepted

Available online

Keywords:

Splenocin B

9-Membered dilactone

Anti-inflammatory

Kita-Trost lactonization

Antimycin-class antibiotics

\section{Introduction}

Splenocins were isolated from an organic extract of marinederived Streptomyces strain CNQ431 as potent antiinflammatory antibiotics in 2009, which displayed low nanomolar activity in the suppression of cytokine production by OVA-stimulated splenocytes. ${ }^{1 a}$ Splenocins exhibit inhibitory activities toward not only the production of TH2 cytokines IL-5 and IL-13 but also the production of the dendritic cell-associated cytokins IL-1 and TNF- $\alpha$, which provide great benefits in the treatment of asthma. The structures of splenocins are similar to those of antimycin $\mathrm{A}_{3}(\mathrm{AA})^{2,3}$ and UK-2A, another antibiotic in the antimycin class, which was first isolated in 1996 from a soil sample collected at our campus. ${ }^{4}$ These consist of 9-membered dilactone rings linked via an amide bond to an aromatic acid moiety (Fig. 1); splenocins and AAs have 3-formamidosalicylic moieties, while UK-2A possesses a 3-hydroxy-4-methoxypicolinic moiety.

Splenocin B (1) is reported to be as effective as dexamethasone in inhibiting TH2 cytokine production and is a hybrid molecule combining some structural features of both UK2A and AA; the benzyl group at the $\mathrm{C} 2$ position in $\mathbf{1}$ had not been reported in AAs. In our continuing studies on UK-2A and AAs, ${ }^{5}$ we have been very interested in the structure and biological activities of splenocin B. Herein we report the first total synthesis of splenocin B (1).

* Corresponding author. Tel.: +81-6-6605-2563; fax: +81-6-6605-2522; e-mail: usuki@ sci.osaka-cu.ac.jp (Y. Usuki)

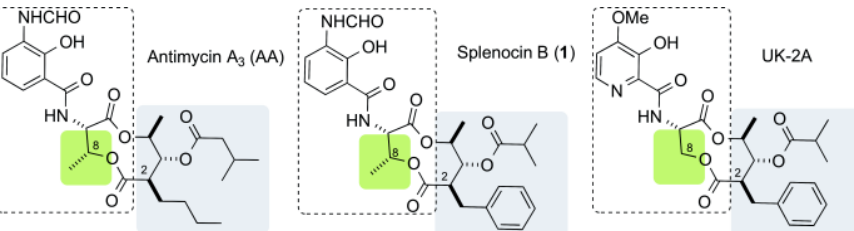

Fig. 1. Structures of Antimycin $A_{3}$, Splenocin $B(1)$ and UK-2A.

\section{Results and disccussion}

Our synthesis of $\mathbf{1}$ commences with the formation of the 3,4dihydroxypentanoic acid derivative $\mathbf{2}$, which was achieved through the Evans aldol reaction between aldehyde $\mathbf{3}$ and $\mathrm{N}$ hydrocinnamoyloxazolidine, as previously reported (Scheme 1). ${ }^{6}$ Condensation of $\mathbf{2}$ with commercially available $\mathrm{N}$-Boc-Lthreonine benzyl ester 4 was conducted in the presence of EDCI. $\mathrm{HCl}$ (3.0 equiv.)-DMAP (0.30 equiv.) to afford 5 in $93 \%$ yield. Removal of the two benzyl groups by hydrogenolysis with $\mathrm{Pd}(\mathrm{OH})_{2}$ in EtOH afforded the cyclization precursor seco acid 6 in $98 \%$ yield. 
<smiles>CC#CCCCCC(C)OC=O</smiles>

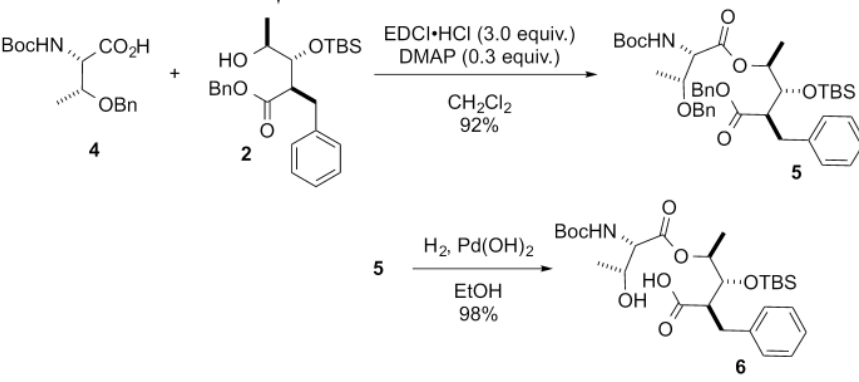

Scheme 1. Synthesis of seco acid 6.

Next, we focused on the construction of a nine-membered dilactone moiety, which appear to be the more difficult due to both enthalpic and entropic factors. The use of 2-methyl-6nitrobenzoic anhydride (MNBA) with a DMAP or DMAPO catalyst, ${ }^{7}$ which was found to be optimal in the previous reports on the synthesis of AAs, ${ }^{3 e, 3 j}$ provided only dimeric 18 -membered tetralactone. After several attempts, including the use of a 2pyridinethiol ester-silver salt, ${ }^{8}$ we executed the Kita-Trost method. ${ }^{9}$ Treatment of $\mathbf{6}$ with ethoxyacetylene (3.0 equiv.) in the presence of $\left[\mathrm{RuCl}_{2}(\mathrm{p} \text {-cymene })\right]_{2} \quad(5 \mathrm{~mol} \%)$ afforded the corresponding ethoxyvinyl ester intermediate 7 . To a solution of CSA $(10 \mathrm{~mol} \%)$ in 1,2-dichloroethane, a dilute solution of 7 in 1,2-dichloroethane was slowly added over $7 \mathrm{~h}$ at $80^{\circ} \mathrm{C}$, followed by stirring for $24 \mathrm{~h}$. The desired reaction proceeded to afford dilactone 8 in $59 \%$ yield (Scheme 2).
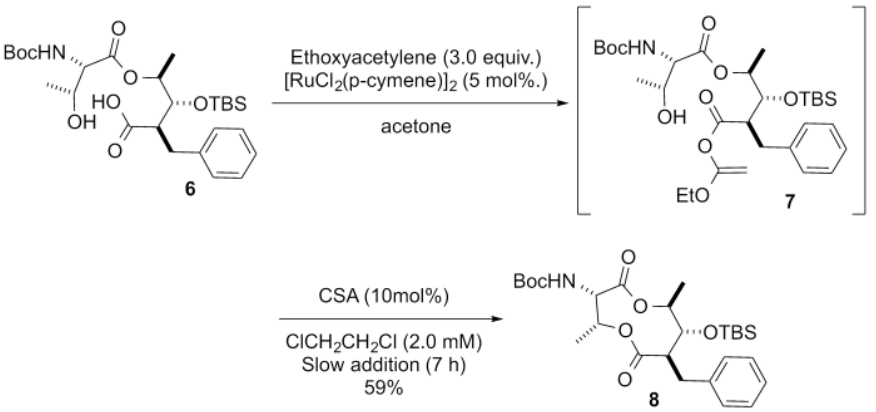

Scheme 2. Construction of the nine-membered dilactone core.

To complete the synthesis of $\mathbf{1}$, the TBS group was removed by treatment of $\mathbf{8}$ with an HF. pyridine complex in a mixture of pyridine and THF to afford 9, which was condensed with isobutyric acid in the presence of EDCI. $\mathrm{HCl}$ (3.0 equiv.)-DMAP (0.30 equiv.) to afford 10 (Scheme 3 ). The $N$-Boc moiety was removed with TFA in dichloromethane to afford 11. Amide formation of $\mathbf{1 1}$ with acid $\mathbf{1 2}$ was achieved in DMF with $\mathrm{EDCl} \cdot \mathrm{HCl}, \mathrm{HOBt}$, and $\mathrm{NMM}$ in $91 \%$ yield. Hydrogenolysis of 13 with $\mathrm{Pd}(\mathrm{OH})_{2}$ in EtOH afforded splenocin B (1) in $98 \%$ yield. The spectral data of synthetic 1 were identical to those reported for the natural sample. The optical rotation of synthetic $1\left([\alpha]_{\mathrm{D}}+\right.$ $24.8, \mathrm{c} 0.11, \mathrm{CHCl}_{3}$ ) was in agreement with that of the natural product $\left([\alpha]_{\mathrm{D}}+21.2, \mathrm{c} 0.01, \mathrm{CHCl}_{3}\right){ }^{1 \mathrm{a}}$

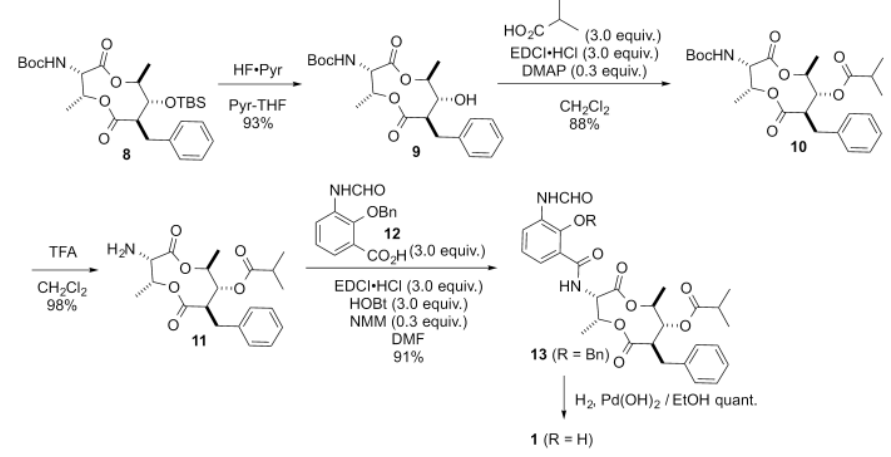

Scheme 3. Final steps in the synthesis of $\mathbf{1}$.

\section{Conclusions}

The total synthesis of splenocin B (1) has been achieved via macrolactonization using ethoxyvinyl ester as a key reagent for furnishing the 9-membered dilactone ring skeleton. Investigations into the application of the developed protocol to the structure-activity relationship are currently underway in our laboratory.

\section{Experimental section}

\subsection{General remarks}

Unless mentioned otherwise, ${ }^{1} \mathrm{H}$ and ${ }^{13} \mathrm{C}$ NMR spectra were recorded on a JEOL JNM-LA 300 (300 and $75 \mathrm{MHz}$ ), a JEOL JNM-LA 400 (400 and $100 \mathrm{MHz}$ ), a Bruker AVANCE 300 (300 and $75 \mathrm{MHz}$ ), or a Bruker AVANCE III 600 (600 and $150 \mathrm{MHz}$ ). Data are reported as follows: chemical shift, multiplicity $(\mathrm{s}=$ singlet, $\mathrm{d}=$ doublet, $\mathrm{t}=$ triplet, $\mathrm{q}=$ quartet, $\mathrm{m}=$ multiplet, and $\mathrm{br}$ = broad), coupling constant in $\mathrm{Hz}$, integration. Coupling constants were determined directly from ${ }^{1} \mathrm{H}$ and ${ }^{13} \mathrm{C}$ NMR spectra. The chemical shifts are reported in $\delta(\mathrm{ppm})$ values relative to $\mathrm{CHCl}_{3}\left(\delta 7.26 \mathrm{ppm}\right.$ for ${ }^{1} \mathrm{H}$ NMR and $\delta 77.0 \mathrm{ppm}$ for ${ }^{13} \mathrm{C}$ NMR) and $\mathrm{Me}_{4} \mathrm{Si}$ ( $\delta 0.00 \mathrm{ppm}$ for ${ }^{1} \mathrm{H}$ NMR). Mass spectra were obtained on a JEOL JMS-700T (EI, CI, FAB) or a Bruker solariX (ESI) spectrometer. IR spectra were recorded on a JASCO FT/IR-4600 spectrometer. Optical rotations were measured on a PERKIN-ELMER 241 polarimeter with a path length of $1 \mathrm{dm}$ or on a JASCO P-1030 with a path length of 0.1 $\mathrm{dm}$ at ambient temperature; the concentrations are reported in $\mathrm{g}$ $\mathrm{dL}^{-1}$. Melting points were determined on a Yanaco MP-I3 micro melting point apparatus and the thermometer was used without correction. All air- and moisture-sensitive reactions were conducted in a flame-dried, argon-flushed, two-necked flask sealed with a rubber septa, and dry solvents and reagents were introduced using a syringe. Tetrahydrofuran (THF) was freshly distilled under an argon atmosphere from sodium benzophenone ketyl. Dichloromethane $\left(\mathrm{CH}_{2} \mathrm{Cl}_{2}\right)$ was freshly distilled from phosphoric pentoxide $\left(\mathrm{P}_{2} \mathrm{O}_{5}\right)$. Flash column chromatography was conducted on a Kanto Chemical silica gel $60 \mathrm{~N}$ (spherical, neutral, 40-50 $\mu \mathrm{m}$ ), and pre-coated Merck silica gel plates (Art5715 Kieselgel $60 \mathrm{~F}_{254}, 0.25 \mathrm{~mm}$ ) were used for thin-layer chromatography (TLC). TLC visualization was accompanied by the use of a UV lamp $(254 \mathrm{~nm})$ or a charring solution (ethanolic $p$-anisaldehyde, ethanolic phosphomolybdic acid).

\section{2. $(2 R, 3 R, 4 S)-2-B e n z y l-4-[(2 S, 3 R)-3$-benzyloxy-2-tert- butoxycarbonylamino-propionyloxy]-3-(tert-butyl-dimethyl- silanyloxy)-pentanoic acid benzyl ester (5)}

To a stirred, cooled $\left(0{ }^{\circ} \mathrm{C}\right)$ solution of freshly prepared $2^{6 \mathrm{~b}}$ (454 mg, $1.06 \mathrm{mmol})$ and $N$-Boc-L-Thr (OBn) 4 (989 mg, 3.20 mmol) in $\mathrm{CH}_{2} \mathrm{Cl}_{2}(8 \mathrm{~mL})$, DMAP $(39 \mathrm{mg}, 0.32 \mathrm{mmol})$ and EDCI - $\mathrm{HCl}(613 \mathrm{mg}, 3.20 \mathrm{mmol})$ were added successively. After 
stirring overnight at $0{ }^{\circ} \mathrm{C}$ to $\mathrm{rt}$, the resulting mixture was diluted with $\mathrm{Et}_{2} \mathrm{O}$-hexane $(1: 2,30 \mathrm{~mL})$ and filtered through a short-pass silica gel column. The filtrate was concentrated and purified by silica gel column chromatography (EtOAc-hexane) to provide $\mathbf{5}$ (614 $\mathrm{mg}, 0.975 \mathrm{mmol}, 92 \%)$ as a clear oil. $[\alpha]_{\mathrm{D}}=+6.7(\mathrm{c}=1.3$, $\mathrm{MeOH}) ;{ }^{1} \mathrm{H} \mathrm{NMR}\left(600 \mathrm{MHz}, \mathrm{CDCl}_{3}\right) \delta 0.05(\mathrm{~s}, 3 \mathrm{H}), 0.070(\mathrm{~s}$, $3 \mathrm{H}), 0.92(\mathrm{~s}, 9 \mathrm{H}), 1.23(\mathrm{~d}, J=6.7 \mathrm{~Hz}, 6 \mathrm{H}), 1.43(\mathrm{~s}, 9 \mathrm{H}), 2.78$ $2.84(\mathrm{~m}, 2 \mathrm{H}), 3.09-3.16(\mathrm{~m}, 1 \mathrm{H}), 4.01-4.04(\mathrm{~m}, 1 \mathrm{H}), 4.09-4.14$ $(\mathrm{m}, 1 \mathrm{H}), 4.31(\mathrm{dd}, J=9.8,2.0 \mathrm{~Hz}, 1 \mathrm{H}), 4.36(\mathrm{~d}, J=11.7 \mathrm{~Hz}, 1 \mathrm{H})$, $4.50(\mathrm{~d}, J=11.7 \mathrm{~Hz}, 1 \mathrm{H}), 4.89-4.95(\mathrm{~m}, 1 \mathrm{H}), 4.92(\mathrm{~s}, 2 \mathrm{H}), 5.30$ $(\mathrm{d}, J=9.8 \mathrm{~Hz}, 1 \mathrm{H}), 7.03(\mathrm{~d}, J=7.3 \mathrm{~Hz}, 1 \mathrm{H}), 7.04(\mathrm{~d}, J=5.3 \mathrm{~Hz}$, $1 \mathrm{H}), 7.10(\mathrm{~d}, J=6.7 \mathrm{~Hz}, 2 \mathrm{H}), 7.18(\mathrm{t}, J=6.8 \mathrm{~Hz}, 2 \mathrm{H}), 7.18(\mathrm{t}, J=$ $7.3 \mathrm{~Hz}, 1 \mathrm{H}), 7.18-7.20(\mathrm{~m}, 1 \mathrm{H}), 7.20-7.26(\mathrm{~m}, 4 \mathrm{H}), 7.26-7.29$ $(\mathrm{m}, 1 \mathrm{H}), 7.33(\mathrm{t}, \mathrm{J}=7.1 \mathrm{~Hz}, 2 \mathrm{H}) ;{ }^{13} \mathrm{C} \mathrm{NMR}\left(150 \mathrm{MHz}, \mathrm{CDCl}_{3}\right) \delta$ $-4.52,-4.05,14.28,16.27,18.31,25.94,28.27,35.23,52.14$, $58.35,66.44,70.87,74.09,74.89,75.15,79.73,126.31,127.63$, 127.72, 128.07, 128.27, 128.38, 128.41, 128.49, 128.87, 135.33, 137.85, 139.03, 155.79, 170.37, 173.00; IR(KBr) $v_{\max } 2931,1781$ 1722, 1497, 1171, 778, 748, $699 \mathrm{~cm}^{-1}$; HRFABMS calcd for $\mathrm{C}_{41} \mathrm{H}_{58} \mathrm{NO}_{8} \mathrm{Si}[\mathrm{M}+\mathrm{H}]^{+}$720.3931: found 720.3929 . $3.77(\mathrm{t}, \mathrm{J}=9.2 \mathrm{~Hz}, 1 \mathrm{H}), 4.70-4.90(\mathrm{~m}, 2 \mathrm{H}), 5.20(\mathrm{~d}, J=7.6 \mathrm{~Hz}$, $1 \mathrm{H}), 5.32-5.45(\mathrm{~m}, 1 \mathrm{H}), 7.12(\mathrm{~d}, J=7.3 \mathrm{~Hz}, 2 \mathrm{H}), 7.11-7.31(\mathrm{~m}$, $3 \mathrm{H}) ;{ }^{13} \mathrm{C}$ NMR $\left(100 \mathrm{MHz}, \mathrm{CDCl}_{3}\right) \delta-3.17,-3.14,14.60,18.68$, $18.99,25.93,28.15,35.48,54.67,55.30,71.32,76.94,77.54$, $126.42,128.40,128.85,138.84,154.93,170.68,173.77$; IR(KBr) $v_{\max } 3367,1739,1709,1521,1454,1361,1249,1169,1095,780$ $\mathrm{cm}^{-1}$; HRFABMS calcd for $\mathrm{C}_{27} \mathrm{H}_{44} \mathrm{NO}_{7} \mathrm{Si}[\mathrm{M}+\mathrm{H}]^{+}$522.2887: found 522.2880 .

\section{5. (3S, 4R, 7R, 8R, 9S)-(7-Benzyl-8-hydroxy-4,9-dimethyl-} 2,6-dioxo-[1,5]dioxonan-3-yl)-carbamic acid tert-butyl ester (9)

Dilactone 8 (104 mg, $0.200 \mathrm{mmol})$ was treated with (HF.pyridine complex)-pyridine-THF (5:6:8, $3.2 \mathrm{~mL}$ ) at $\mathrm{rt}$ until the starting material disappeared. The mixture was diluted with EtOAc, poured into stirred saturated aq. $\mathrm{NaHCO}_{3}$, and extracted with EtOAc $(2 \mathrm{x})$. The combined extracts were washed with brine, dried over $\mathrm{Na}_{2} \mathrm{SO}_{4}$, concentrated, and purified by silica gel column chromatography (EtOAc-hexane) to provide $\mathbf{9}(75.7 \mathrm{mg}$, $0.186 \mathrm{mmol}, 93 \%)$ as a clear oil. $[\alpha]_{\mathrm{D}}=+55\left(\mathrm{c}=0.29, \mathrm{CHCl}_{3}\right) ;{ }^{1} \mathrm{H}$ NMR $\left(400 \mathrm{MHz}, \mathrm{CDCl}_{3}\right) \delta 1.15(\mathrm{~d}, J=6.6 \mathrm{~Hz}, 3 \mathrm{H}), 1.43(\mathrm{~s}, 9 \mathrm{H})$, $1.45(\mathrm{~d}, J=6.5 \mathrm{~Hz}, 3 \mathrm{H}), 2.66(\mathrm{ddd}, J=10.8,9.6,3.6 \mathrm{~Hz} 1 \mathrm{H})$, $2.98(\mathrm{dd}, J=13.4,10.8 \mathrm{~Hz}, 1 \mathrm{H}), 3.18(\mathrm{dd}, J=13.4,3.6 \mathrm{~Hz}, 1 \mathrm{H})$, $3.69(\mathrm{t}, J=9.6 \mathrm{~Hz}, 1 \mathrm{H}), 4.75-4.87(\mathrm{~m}, 2 \mathrm{H}), 5.17-5.27(\mathrm{~m}, 1 \mathrm{H})$, $5.41(\mathrm{~m}, 1 \mathrm{H}), 7.14-7.29(\mathrm{~m}, 5 \mathrm{H}) ;{ }^{13} \mathrm{C}$ NMR $\left(150 \mathrm{MHz}, \mathrm{CDCl}_{3}\right) \delta$ $14.38,18.38,28.24,31.58,35.06,53.90,54.32,71.06,75.95$, $80.36,128.48,128.53,128.84,138.67,154.85,170.65,172.96$; $\operatorname{IR}(\mathrm{KBr}) v_{\max } 3396,1687,1544,1509,1135,1044,698 \mathrm{~cm}^{-1}$; HRFABMS calcd for $\mathrm{C}_{21} \mathrm{H}_{28} \mathrm{NO}_{7}[\mathrm{M}-\mathrm{H}]^{-}$406.1866: found 406.1861.

\subsection{Isobutyric acid $(3 S, 4 R, 7 R, 8 R, 9 S)$-[7-benzyl-3-tert- butoxycarbonylamino-4,9-dimethyl-2,6-dioxo-[1,5]dioxonan- 8-yl] ester (10)}

To a stirred, cooled $\left(0^{\circ} \mathrm{C}\right)$ solution of $9(17.8 \mathrm{mg}, 44 \mu \mathrm{mol})$ and isobutyric acid $(18 \mu \mathrm{L})$ in $\mathrm{CH}_{2} \mathrm{Cl}_{2}(0.5 \mathrm{~mL})$ was added DMAP $(2 \mathrm{mg}, 16 \mu \mathrm{mol})$ and $\mathrm{EDCI} \cdot \mathrm{HCl}(36 \mathrm{mg}, 18 \mu \mathrm{mol})$ successively. After stirring overnight at $\mathrm{rt}$, the resulting mixture was poured into $\mathrm{H}_{2} \mathrm{O}$ and extracted with EtOAc (3x). The combined extracts were washed with $\mathrm{H}_{2} \mathrm{O}(2 \mathrm{x})$ and brine, dried over $\mathrm{Na}_{2} \mathrm{SO}_{4}$, concentrated, and purified by silica gel column chromatography (20\% EtOAc-hexane) to give $\mathbf{1 0}$ as a white solid (18.4 mg, $39 \mu \mathrm{mol}, 88 \%)$. Mp $122{ }^{\circ} \mathrm{C} ;[\alpha]_{D}=+57(\mathrm{c}=0.11$, $\left.\mathrm{CHCl}_{3}\right) ;{ }^{1} \mathrm{H} \mathrm{NMR}\left(400 \mathrm{MHz}, \mathrm{CDCl}_{3}\right) \delta 1.15(\mathrm{~d}, J=6.6 \mathrm{~Hz}, 3 \mathrm{H})$, $1.217(\mathrm{~d}, J=6.8 \mathrm{~Hz}, 3 \mathrm{H}), 1.224(\mathrm{~d}, J=6.8 \mathrm{~Hz}, 3 \mathrm{H}), 1.30(\mathrm{~d}, J=$ $6.3 \mathrm{~Hz}, 3 \mathrm{H}), 1.43$ (s, 9H), 2.59 (sept, $J=6.8 \mathrm{~Hz}, 1 \mathrm{H}$ ), 2.66 (dd, $J$ $=3.2,13.4 \mathrm{~Hz}, 1 \mathrm{H}), 2.85(\mathrm{ddd}, J=11.6,10.0,3.2 \mathrm{~Hz}, 1 \mathrm{H}), 2.97$ $(\mathrm{dd}, J=11.6,13.4 \mathrm{~Hz}, 1 \mathrm{H}), 4.83-4.97(\mathrm{~m}, 2 \mathrm{H}), 5.17(\mathrm{t}, J=10.0$ $\mathrm{Hz}, 1 \mathrm{H}), 5.16-5.19(\mathrm{~m}, 1 \mathrm{H}), 5.42-5.45(\mathrm{~m}, 1 \mathrm{H}), 7.07-7.30(\mathrm{~m}$, $5 \mathrm{H}) ;{ }^{13} \mathrm{C}$ NMR $\left(100 \mathrm{MHz}, \mathrm{CDCl}_{3}\right) \delta 14.45,17.77,18.86,28.15$, $34.03,34.45,51.75,54.27,71.28,74.08,75.25,126.59,128.54$, $128.76,138.15,154.62,170.67,171.99,175.73$; IR(KBr) $v_{\max }$ 3368, 1741, 1699, 1510, 1369, 1149, 743, $704 \mathrm{~cm}^{-1}$; HRFABMS calcd for $\mathrm{C}_{25} \mathrm{H}_{36} \mathrm{NO}_{8}[\mathrm{M}+\mathrm{H}]^{+}$478.2441: found 478.2438.

\subsection{Isobutyric acid $(3 S, 4 R, 7 R, 8 R, 9 S)$-[3-amino-7-benzyl- 4,9-dimethyl-2,6-dioxo-[1,5]dioxonan-8-yl] ester (11)}

To a solution of $\mathbf{1 0}(9.2 \mathrm{mg}, 19 \mu \mathrm{mol})$ in $\mathrm{CH}_{2} \mathrm{Cl}_{2}(1 \mathrm{~mL})$, trifluoroacetic acid $(0.5 \mathrm{~mL}, 0.759 \mathrm{mmol})$ was added dropwise. After stirring at $\mathrm{rt}$ for $2 \mathrm{~h}$, the resulting mixture was concentrated, diluted with saturated aq. $\mathrm{NaHCO}_{3}$, and extracted with EtOAc (2x). The combined extracts were washed with brine, dried over $\mathrm{Na}_{2} \mathrm{SO}_{4}$, and concentrated to produce crude $11(8.5 \mathrm{mg}, 23 \mu \mathrm{mol}$, $98 \%)$ as a white solid. This was used for the next reaction without further purification. Mp $124{ }^{\circ} \mathrm{C} ;[\alpha]_{D}=+0.54(\mathrm{c}=0.56$, $\mathrm{MeOH}) ;{ }^{1} \mathrm{H}$ NMR (400 MHz, $\left.\mathrm{CDCl}_{3}\right) \delta 1.20(\mathrm{~d}, J=6.3 \mathrm{~Hz}, 3 \mathrm{H})$, 
$1.218(\mathrm{~d}, J=6.8 \mathrm{~Hz}, 3 \mathrm{H}), 1.224(\mathrm{~d}, J=6.8 \mathrm{~Hz}, 3 \mathrm{H}), 1.29(\mathrm{~d}, J=$ $6.3 \mathrm{~Hz}, 3 \mathrm{H}), 2.58(\mathrm{sept}, J=6.8 \mathrm{~Hz}, 1 \mathrm{H}), 2.65(\mathrm{dd}, J=2.8,13.6$ $\mathrm{Hz}, 1 \mathrm{H}), 2.88(\mathrm{ddd}, J=11.2,10.0,2.8 \mathrm{~Hz}, 1 \mathrm{H}), 2.99(\mathrm{dd}, J=$ $113.6,11.2 \mathrm{~Hz}, 1 \mathrm{H}), 4.13(\mathrm{~d}, J=8.1 \mathrm{~Hz}, 1 \mathrm{H}), 4.82(\mathrm{dq}, J=10.0$, $6.3 \mathrm{~Hz}, 1 \mathrm{H}), 5.13(\mathrm{t}, J=10.0 \mathrm{~Hz}, 1 \mathrm{H}), 5.17-5.20(\mathrm{~m}, 1 \mathrm{H}), 7.11-$ $7.30(\mathrm{~m}, 5 \mathrm{H}) ;{ }^{13} \mathrm{C} \mathrm{NMR}\left(150 \mathrm{MHz}, \mathrm{CDCl}_{3}\right) \delta 13.00,18.93$, $18.96,29.69,34.11,34.56,51.64,53.82,72.08,73.86,75.26$, $126.50,128.49,128.73,138.28,171.51,174.14,175.75 ; \mathrm{IR}(\mathrm{KBr})$ $6 v_{\max } 3397,2983,1741,1454,1177,702 \mathrm{~cm}^{-1}$; HRFABMS calcd 7 for $\mathrm{C}_{20} \mathrm{H}_{28} \mathrm{NO}_{6}[\mathrm{M}+\mathrm{H}]^{+}$378.1916: found 378.1921.

8 4.8. Isobutyric acid $(3 S, 4 R, 7 R, 8 R, 9 S)$-7-benzyl-3-(39 formylamino-2-benzyloxy-benzoylamino)-4,6-dimethyl-2,6$(\mathrm{dd}, J=7.6,7.6 \mathrm{~Hz}, 1 \mathrm{H}), 5.62$ (quint, $J=6.9 \mathrm{~Hz}, 1 \mathrm{H}), 6.91(\mathrm{t}, J=$ $8.1 \mathrm{~Hz}, 1 \mathrm{H}), 6.99(\mathrm{~d}, J=7.7 \mathrm{~Hz}, 1 \mathrm{H}), 7.13(\mathrm{~d}, J=7.2 \mathrm{~Hz}, 2 \mathrm{H})$, $7.19(\mathrm{~d}, J=7.4 \mathrm{~Hz}, 1 \mathrm{H}), 7.20(\mathrm{~d}, J=8.4 \mathrm{~Hz}, 1 \mathrm{H}), 7.24(\mathrm{t}, J=7.5$ $\mathrm{Hz}, 2 \mathrm{H}), 7.89(\mathrm{~s}, 1 \mathrm{H}), 8.50(\mathrm{~s}, 1 \mathrm{H}), 8.54(\mathrm{~d}, J=7.2 \mathrm{~Hz}, 1 \mathrm{H})$, $12.60(\mathrm{~s}, 1 \mathrm{H}) ;{ }^{13} \mathrm{C}$ NMR $\left(150 \mathrm{MHz}, \mathrm{CDCl}_{3}\right) \delta 14.71,17.81$, $18.95,34.11,34.53,51.91,53.46,70.92,74.78,75.06,112.54$, $118.94,120.08,124.80,126.65,127.44,128.55,128.72,137.90$, $150.62,159.06,169.33,170.07,171.94,175.65 ; \mathrm{IR}(\mathrm{KBr}) v_{\max }$ 3352, 2932, 1737, 1524, 1376, 1151, 749, $703 \mathrm{~cm}^{-1}$; HRESIMS calcd. for $\mathrm{C}_{28} \mathrm{H}_{31} \mathrm{~N}_{2} \mathrm{O}_{9}[\mathrm{M}-\mathrm{H}]^{-}$539.2035: found 539.2035.

\section{Acknowledgments}

This paper is dedicated to the memory of late Professor Kozo Shibata, Osaka City University. We thank Dr Matsumi Doe, Analytical Division, Osaka City University for NMR measurements.

\section{References and notes}

1. Isolation and structure elucidation of splenocin's. (a) Fenical, W.; Strangman, W. K.; Kwon, H. K.; Broide, D.; Jensen, P. R. J. Med. Chem. 2009, 52, 2317-2327; Biosynthesis of splenocin's. (b) Chang, C.; Huang, R.; Yan, Y.; Ma, H.; Dai, Z.; Zhang, B.; Deng, Z.; Liu, W.; Qu, X. J. Am. Chem. Soc. 2015, 137, 4183-4190.

2. Isolation and structure elucidation of AA. (a) Leben, C.; Keitt, G. W. Phytopathology 1948, 38, 899-906; (b) Dunshee, B. R.; Leben, C.; Keitt, G. W.; Strong, F. M. J. Am. Chem. Soc. 1949, 71, 2436-2437.

3. Total synthesis of AA. (a) Kinoshita, M.; Wada, M.; Aburagi, S.; Umezawa, S. J. Antibiot. 1971, 24, 724-726; (b) Kinoshita, M.; Aburaki, S.; Wada, M.; Umezawa, S. Bull. Chem. Soc. Jpn. 1973, 46, 1279-1287; (c) Tsunoda, T.; Nishii, T.; Yoshizuka, M.; Yamasaki, C.; Suzuki, T.; Ito, S. Tetrahedron Lett. 2000, 41 7667-7671; (d) Nishii, T.; Suzuki, S.; Yoshida, K.; Araki, K.; Tsunoda, T. Tetrahedron Lett. 2003, 44, 7829-7832; (e) Wu, Y.; Yang, Y. J. Org. Chem. 2006, 71, 4296-4301; (f) Chakraborty, T. K.; Chattopadhyay, A. K.; Ghosh, S. Tetrahedron Lett. 2007, 48, 1139-1142; (g) Hu, Z.; Jiang, X.; Han, W. Tetrahedron Lett. 2008, 49, 5192-5195; (h) Inai, M.; Nishii, T.; Tanaka, A.; Kaku, H.; Horikawa, M.; Tsunoda, T. Eur. J. Org. Chem. 2011, 27192729; (i) Iijima, Y.; Kimata, O.; Decharin, S.; Masui, H.; Hirose, Y.; Takahashi, T. Eur. J. Org. Chem. 2014, 4725-4732; (j) Janetzko, J.; Batey, R. A. J. Org. Chem. 2014, 79, 7415-7424.

4. (a) Ueki, M.; Abe, K.; Hanafi, M.; Shibata, K.; Tanaka, T.; Taniguchi, M. J. Antibiot. 1996, 49, 639-643; (b) Hanafi, M.; Shibata, K.; Ueki, M.; Taniguchi, M. J. Antibiot. 1996, 49, 1226 1231.

5. (a) Usuki, Y.; Goto, K.; Kiso, T.; Tani, K.; Ping, X.; Fujita, K.-I. Iio, H.; Taniguchi, M. J. Antibiot. 2002, 55, 607-610; (b) Fujita, K.-I.; Kiso, T.; Usuki, Y.; Tanaka, T.; Taniguchi, M. J. Antibiot. 2004, 57, 687-690; (c) Usuki, Y.; Mitomo, M.; Adachi, N.; Ping, X.; Fujita, K.-I.; Sakanaka, O.; Iinuma, K.; Iio, H.; Taniguchi, M. Bioorg. Med. Chem. Lett. 2005, 15, 2011-2014.

6. (a) Shimano, M.; Shibata, T.; Kamei, N. Tetrahedron Lett. 1998, 39, 4363-4366; (b) Shimano, M.; Kamei, N.; Shibata, T. Inoguchi, K.; Ito, N.; Ikari, T.; Senda, H. Tetrahedron 1998, 54 , 12745-12774.

7. For a recent review on MNBA, see. Shiina, I. Bull. Chem. Soc. Jpn. 2014, 87, 196-233.

8. For a recent review on macrolactonization, see. Parenty, A.; Moreau, X.; Niel, G.; Campagne, J.-M. Chem. Rev. 2013, 113, PR1-PR40.

9. (a) Kita, Y.; Maeda, H.; Omori, K.; Okuno, T.; Tamura, Y. Synlett 1993, 273-274; (b) Trost, B. M.; Chisholm, J. D. Org. Lett. 2002, 4, 3743-3745; (c) Trost, B. M.; Harrington, P. E.; Chisholm, J. D.; Wrobleski, S. T. J. Am. Chem. Soc. 2005, 127 , 13598-13610; (d) Ohba, Y.; Takatsuji, M.; Nakahara, K.; Fujioka, H.; Kita, Y. Chem. Eur. J. 2009, 3526-3537.

10. ${ }^{1} \mathrm{H}$ and ${ }^{13} \mathrm{C}$ NMR spectra indicated that it existed as a mixture of two rotamers.

11. Mp was not reported in ref $1 \mathrm{a}$. 


\section{Graphical Abstract}

To create your abstract, type over the instructions in the template box below.

Fonts or abstract dimensions should not be changed or altered.

Total synthesis of splenocin $B$, a potent Leave this area blank for abstract info. inhibitor of the pro-inflammatory cytokine from marine-derived streptomyces sp.

Ken-ichi Yoshida, Minako Ijiri, Hideo Iio, and Yoshinosuke Usuki*

Graduate School of Science, Osaka City University

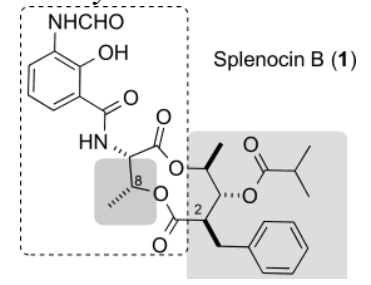

